\title{
Diversité, Distribution Et Statut De Conservation Des Primates Dans Les Reliques De Forêts Dans La Région Du Tonkpi, À L'ouest De La Côte d'Ivoire
}

\author{
Bamba Kramoko, Doctorant-chercheur \\ Béné Jean-Claude Koffi, Enseignant-chercheur \\ Célestin Yao Kouakou, Enseignant-chercheur \\ Kouamé Antoine N'Guessan, Enseignant-chercheur \\ Victorien Kouakou Claude, Doctorant-chercheur
}

Université Jean Lorougnon Guédé, UFR Environnement, Côte d'Ivoire

doi: 10.19044/esj.2017.v13n26p20 URL:http://dx.doi.org/10.19044/esj.2017.v13n26p20

\begin{abstract}
Tropical forests constitute the main part of the non-human primate's habitat but they are more and more fragmented because of anthropic activities. In Ivory Coast, the present forest cover includes only some fragments more or less important. The knowledge of the primate's diversity and their distribution in these relics of forest constitute some hopeful elements for their conservation. This study has been carried out in two classified forests and a community forest of the Tonkpi area in the West of Ivory Coast. It aims first, to assess the species of primates, then to highlight their spatial distributions and at last, determine their conservation status and the threats there are facing. The methodology used consisted firstly, on interview among hunters and older living around the forests to have an overall view of the monkey species in the area and the forests that housing them. Next, foot surveys have been organized in the identified forests during the interview. At the end of this study, seven species of primates belonging to West African forests have been confirmed in these forest relics base on observation of nests, vocalizations and visual signs. The majority of these species are observed at the periphery of forests mainly in mountain and flooded areas apart from Galagoïdes thomasi which is more abundant even in fallow land. Among those species, one (Piliocolobus badius) is listed Endangered (EN) and Colobus polykomos is considered vulnerable (VU) by IUCN; and five others are least Concern (LC). The sustainable conservation of those primates is threatened by agriculture, traditional and industrial mining, hunting and logging. The existence in those forest relics of primate species with conservation concerned justify their value for the sustainable
\end{abstract}


conservation of those primates and the need to reinforce measure of protection.

Keywords: Primates, conservation, threat, forest relics, Ivory Coast

\section{Résumé}

Les forêts tropicales constituent l'essentiel de l'habitat des primates non-humains, mais elles sont de plus en plus fragmentées du fait des activités anthropiques. En Côte d'Ivoire, le couvert forestier actuel se résume en des fragments plus ou moins importants. La connaissance de la diversité des primates et de leur distribution dans ces reliques de forêt s'avère donc importante en vue d'apporter des mesures de conservation. Cette étude a été réalisée dans deux forêts classées et une forêt villageoise de la région du Tonkpi dans l'ouest de la Côte d'Ivoire. Elle vise à inventorier les espèces de primates puis à mettre en relief leurs distributions spatiales et enfin déterminer leur statut de conservation ainsi que les menaces qui pèsent sur elles. La méthodologie utilisée a consisté d'abord en des enquêtes auprès des populations riveraines de ces fragments de forêt pour avoir une idée générale des espèces de singes dans la région et les forêts qui les abritent. Ensuite des prospections pédestres ont été organisées dans les forêts identifiées au cours des enquêtes. A l'issue de cette étude, sept espèces de primates caractéristiques des forêts Ouest africaines ont été confirmées dans ces reliques forestières à partir des observations directes et indirectes. Ces espèces sont rencontrées pour la plupart en périphérie des forêts, notamment dans les forêts sur les montagnes et dans des zones inondées à l'exception de Galagoïdes thomasi qui est plus abondante même dans les jachères. Parmi ces espèces, une (Piliocolobus badius) est classée en Danger (EN), une (Colobus polykomos) autre dans la catégorie Vulnérable (VU) et cinq (5) relèvent de la catégorie Préoccupation mineure (LC). La conservation durable de ces primates est menacée par l'agriculture, 1'orpaillage, la chasse, les exploitations minières et forestières. La présence dans ces reliques forestières d'espèces de primates dont la protection est d'intérêt mondial démontre leur valeur pour la conservation durable des primates et le besoin d'un renforcement des mesures de protection.

Mots-clés : Primates, conservation, menace, relique forestière, Côte d'Ivoire

\section{Introduction}

La biodiversité atteint son maximum dans les régions tropicales dont les forêts abritent plus de $50 \%$ de la diversité biologique mondiale avec plusieurs espèces de primates non-humains (Caspary et al., 2001 ; Koné et al., 2014). La Côte d'Ivoire, située en zone tropicale, abrite ainsi, de par la 
diversité de son écosystème, une faune riche et diversifiée. En effet, la quasitotalité des primates des forêts tropicales y est représentée (Zadou et al., 2011 ; Béné et al., 2015). Malheureusement, ces primates sont soumis à de fortes pressions anthropiques susceptibles de remettre en cause leur conservation durable (Caspary et al., 2001 ; Yéo et al., 2013). Les facteurs de pression sur les forêts ivoiriennes, et plus particulièrement sur les primates sont dans l'ordre de leurs importances relatives : la déforestation, l'expansion de l'agriculture, le braconnage, les feux de brousse incontrôlés, l'urbanisation et l'exploitation minière artisanale et/ou semi-industrielle (Koné, 2004 ; Kadjo et al., 2014 ; Béné et al., 2015).

Dans l'objectif d'atténuer la disparition de sa biodiversité, l'Etat ivoirien a procédé à la création de huit parcs nationaux, six réserves forestières et deux cent trente et un (231) forêts classées (Assoa, 2004). Malgré cet acte novateur, la perte des habitats des primates s'accentue toujours au point où les primates se retrouvent de plus en plus confinés dans des fragments de forêts sur le territoire ivoirien (Koné et al., 2008). L'ouest de la Côte d'Ivoire, précisément la région du Tonkpi n'échappe pas à cette triste réalité et le couvert forestier est constitué par des fragments plus ou moins bien conservés. Au nombre de ces fragments, l'on compte trois reliques de forêts dont deux forêts classées (les forêts classées de Krozialé et Goulaleu) et une forêt villageoise. De ces forêts, aucune information n'est disponible sur leur richesse faunique en général, mais particulièrement sur la faune de primates susceptible d'y être rencontrée. Evaluer la richesse spécifique des primates dans ces reliques forestières s'avère primordiale, d'abord pour avoir une idée de l'état de conservation de ces forêts. Ensuite cette étude permettra d'attirer l'attention, des populations riveraines, des gestionnaires des forêts classées et ceux des forêts du domaine rural, de la région du Tonkpi, sur l'importance des fragments de forêts encore bien conservés. D'ailleurs, il est reconnu que les primates jouent un rôle très important de pollinisateurs, de disperseurs de grains, et de prédateurs d'insectes nuisibles (Béné et al., 2007). Cette étude vise, à inventorier les espèces de primates puis à mettre en relief leurs distributions spatiales et enfin déterminer leur statut de conservation ainsi que les menaces qui pèsent sur elles dans ces trois reliques forestières.

\section{Méthodes}

\section{Site d'étude}

Les forêts prospectées sont localisées à l'Ouest de la Côte d'Ivoire dans la région du Tonkpi, plus précisément dans le département de Zouanhounien. Elles s'étendent entre $6^{\circ} 40^{\prime}$ et $7^{\circ} 0^{\prime}$ de latitude Nord et entre $8^{\circ} 20^{\prime}$ et $8^{\circ} 0^{\prime}$ de longitude Ouest. Les forêts classées de Krozialé et Goulaleu ont respectivement une superficie de 9300 hectares et 950 hectares alors que la 
forêt villageoise s'étend sur une superficie de 170 hectares (Figure 1). La pluviométrie de la région du Tonkpi varie entre 1300 et $2400 \mathrm{~mm}$ par an. Deux grandes saisons se relaient dans la zone : la saison pluvieuse qui va d'avril à octobre et la saison sèche, de novembre à mars (Dieulin, 2008). Des séries de bas plateaux s'observent dans la région. On y trouve aussi des zones très accidentées aux contours variant entre des pentes moyennes et des altitudes dépassant parfois les $1000 \mathrm{~m}$ (N'Guessan et al., 2010). L'économie de la population locale est basée sur l'agriculture pratiquée sur des sols de types ferralitiques à fertilité chimique moyenne, des sols hydromorphes dans les bas-fonds et des sols minéraux en région montagneuse (CNRA, 2009).

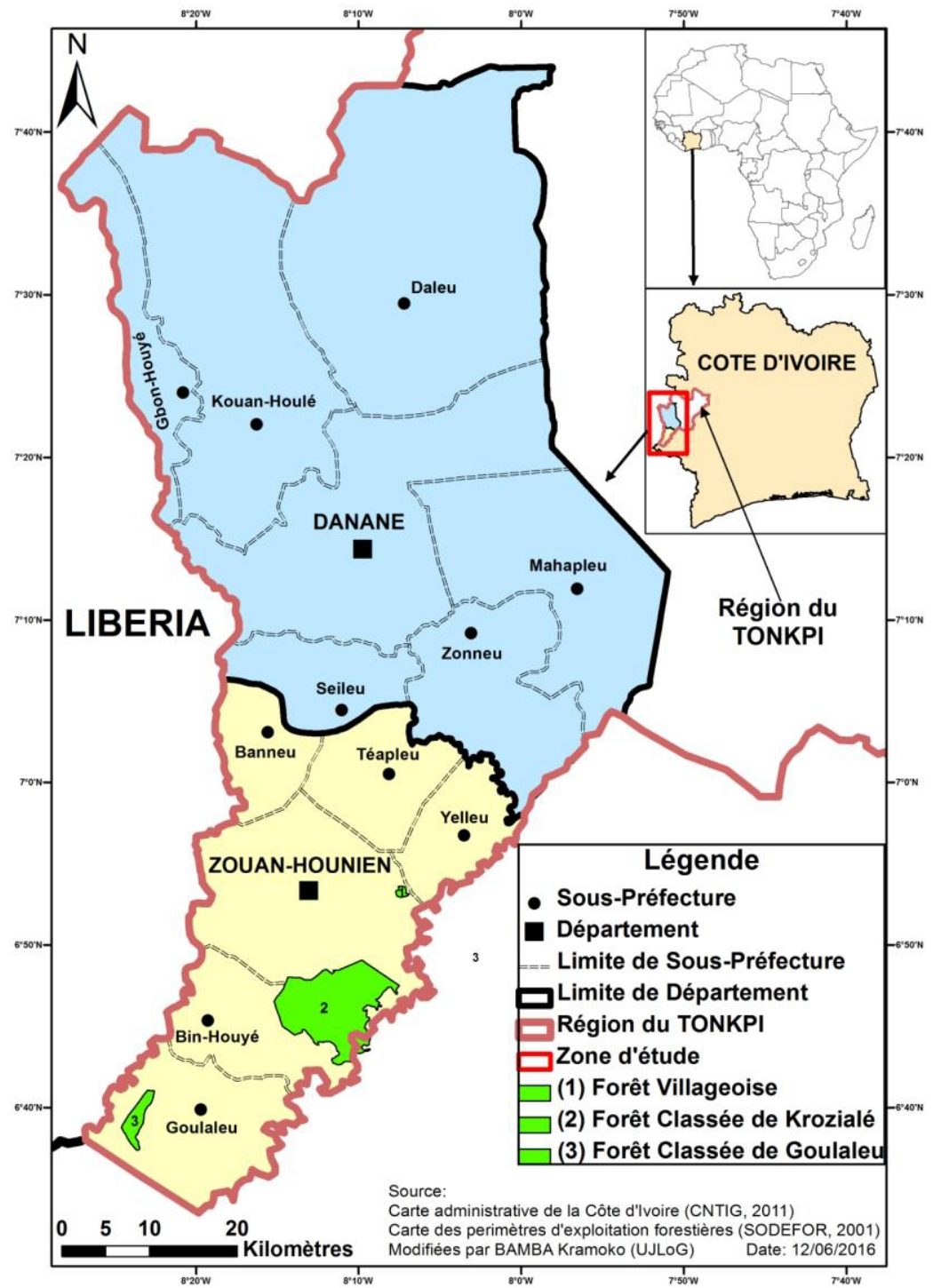

Figure1: Localisation des forêts visitées et de la zone d'étude 


\section{Collecte des données Enquête}

Une enquête auprès des chasseurs et de vieilles personnes ayant des connaissances avérées sur la faune en général et les primates en particulier a été menée dans les villages autour des forêts. Au cours de l'enquête, nous avons montré un échantillon présélectionné de différentes images d'espèces de primates susceptibles d'être présentes dans la région, ainsi que plusieurs autres images de primates qui ne sont pas connus dans la région pour évaluer la fiabilité de l'identification à travers les photos. Cette enquête a permis de dresser une liste des primates qu'on pourrait rencontrer, ensuite à identifier les forêts qui abritent encore ces primates ainsi qu'à évaluer leur statut local de conservation. Un questionnaire a aussi été adressé aux agents de la SODEFOR (gestionnaire des forêts classées) avec pour but (i) d'identifier toutes les forêts classées de la région du Tonkpi, (ii) de connaître les forêts classées potentiellement riches en faune sauvage, (iii) d'avoir une connaissance des primates présents dans la région.

\section{Prospections pédestres}

Les prospections pédestres au cours de cette étude ont été conduites de jour comme de nuit dans les deux forêts classées et dans la forêt villageoise en combinant la méthode des transects linéaires (Kadjo et al., 2014 ; Hoppe-Dominik et al., 2011 ; Buckland et al., 1993) et celle des marches de reconnaissance (RECCE). Ces deux méthodes ont été combinées pour augmenter nos efforts et couvrir les zones de diversité et de densité potentiellement élevées et accroitre nos chances de détecter des espèces menacées. La méthode de transect a consisté à parcourir, au total, quinze (15) lignes droites virtuelles de $600 \mathrm{~m}$ à $3 \mathrm{Km}$ de long à l'intérieur des forêts et positionnées de manière à prospecter les principaux habitats des sites. Lors des parcours des transects linéaires, les déviations ne sont pas autorisées. Quant aux marches de reconnaissances, nous avons suivi une direction prédéterminée, mais qui autorise des déviations au cours des prospections (White et Edwards, 2000). Les recces ont l'avantage de permettre à l'équipe de couvrir plus de zones dans un court laps de temps, tout en ayant un impact minimum sur l'environnement (Walsh et White, 1999; White et Edwards, 2000).

A l'aide du GPS et d'une boussole, nous marchons lentement le long de lignes à une vitesse de 0,5 à $1 \mathrm{~km} / \mathrm{h}$ en vue de collecter des données relatives à la présence des primates. La détection des espèces le long des transects peut être directe ou indirecte. Les observations directes concernent tout contact visuel avec les animaux lors de la marche sur le transect. Les observations indirectes prennent en compte l'ensemble des indices indirects pouvant justifier de façon indubitable la présence des primates sur les sites. 
Ces indices sont généralement constitués de vocalisations. Chaque fois qu'un indice de présence est trouvé, l'équipe d'inventaire s'arrête pour le caractériser et prendre les coordonnées géographiques. Lors des prospections pédestres, nous avons aussi enregistré tous indices d'activités ou de présences anthropiques telles que les pistes, agriculture, orpaillage, chasse etc. Toutes les observations sont reportées sur une fiche d'inventaire pédestre conçue à cet effet.

\section{Analyse des données}

Deux logiciels (PAST (2.17c) et ARC Gis (10.0)) ont été utilisés pour l'analyse des données. Le logiciel PAST (2.17c) a permis d'effectuer les tests statistiques au niveau des différences de fréquences, le calcul de l'abondance numérique, des valeurs d'indice de Shannon et de l'équitabilité. Le logiciel ARC Gis (10.0) a permis la réalisation des cartes de la distribution spatiale des primates inventoriés dans chaque forêt.

\section{Résultats}

\section{Richesse spécifique de primates dans les trois forêts}

Le nombre total d'indice de présence des primates dans les trois forêts est de 875 . La fréquence d'observations des primates dans la forêt classée de Goulaleu est de 42,29\% ( $\mathrm{N}=370)$ dont 73,78\% d'observations directes $(\mathrm{N}=273)$ et $26,22 \%$ d'observations indirectes $(\mathrm{N}=97)$. La fréquence des indices de présence des primates dans la forêt classée de Krozialé est de 34,40\% $(\mathrm{N}=301)$. Parmi ces indices de présence, $74 \%(\mathrm{~N}=$ $223)$ sont directs et $26 \%(\mathrm{~N}=78)$ sont indirects. Dans la forêt villageoise, il y a eu aussi des observations directes $(71,56 \% ; \mathrm{N}=146)$ et des observations indirectes $(28,44 \% ; \mathrm{N}=60)$ sur une fréquence totale d'indice de présence de $23,31 \%(\mathrm{~N}=204)$ en tenant compte de l'ensemble des observations dans les trois reliques forestières pour cette forêt villageoise. Les différences au niveau des fréquences d'observations directes et indirectes des primates dans les trois forêts ne sont pas significatives ( $\mathrm{P}$ value $=0,73>0,05$ ).

La présence de sept espèces de primate a été confirmée à l'issue de ces observations directes et indirectes dans les trois forêts (Figure 2). Galagoïdes thomasi a la plus forte fréquence $(49,44 \% ; \mathrm{N}=433)$. Cette espèce est suivie par Perodictitus potto avec 17,48\% $(\mathrm{N}=153)$. Cercopithecus campbelli a $12,8 \%(\mathrm{~N}=112)$ tandis que Cercopithecus petaurista a une fréquence de $11,66 \%(\mathrm{~N}=102)$. Les espèces rarement observées sont Piliocolobus badius $(3,48 \% ; \mathrm{N}=30)$, Cercopithecus sabaeus $(3,20 \% ; \mathrm{N}=28)$ et Colobus polykomos $(1,94 \% ; \mathrm{N}=17)$.

Toutes ces espèces observées en forêt avaient été mentionnées par les populations lors des enquêtes. Par contre d'autres espèces citées lors de ces enquêtes n'ont pas été confirmées en forêt. Il s'agit du Cercocèbe enfumé (Cercocebus atys) et du Colobe vert (Procolobus verus). 


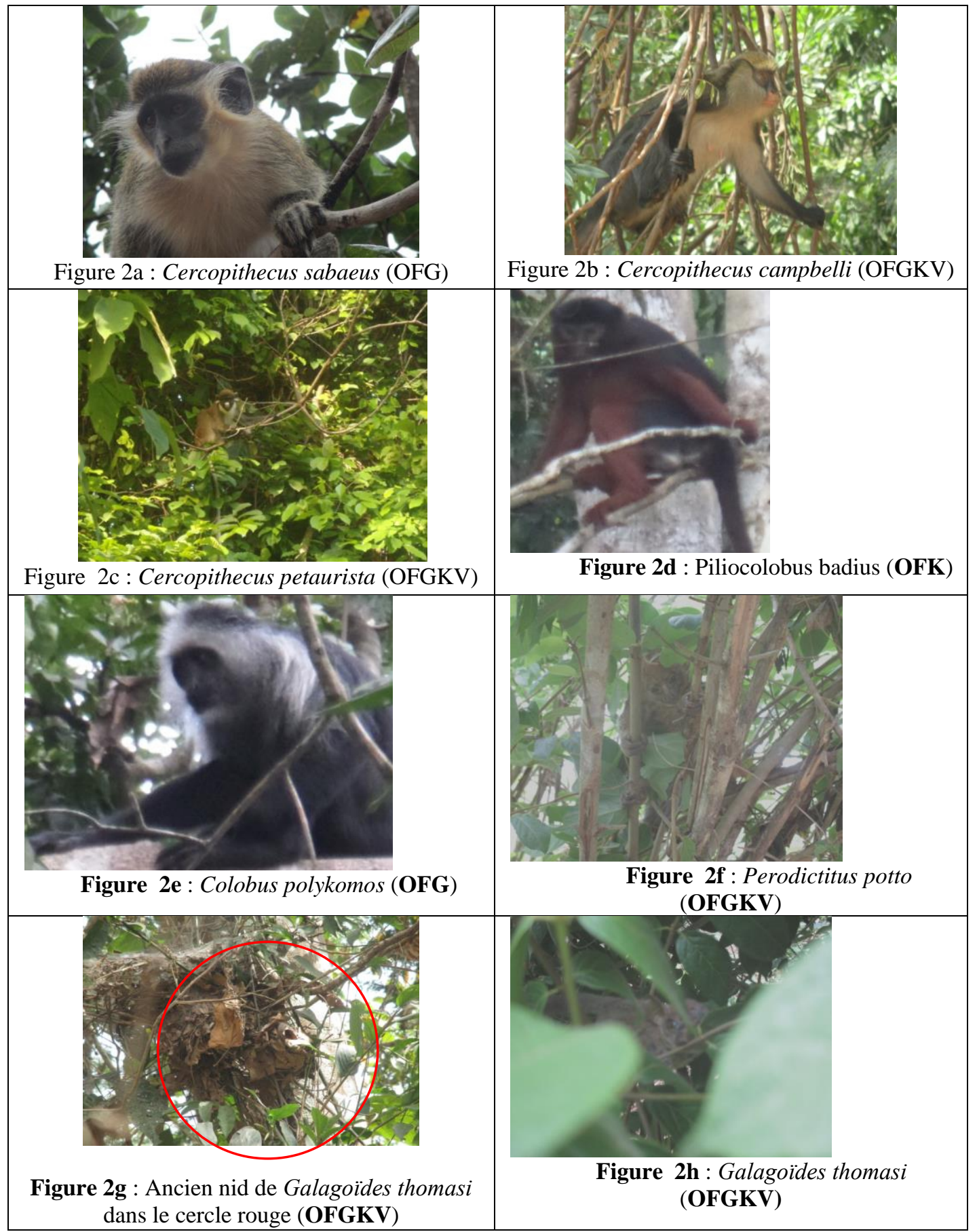

OFG = Observation en Forêt classée de Goulaleu ; OFK = Observation en Forêt classée de

Krozialé ; OFV = Observation en Forêt Villageoise ; OFGKV = Observation en Forêt classées de Goulaleu, Krozialé et en forêt Villageoise

Figure 2: Illustration des indices de présence de primates dans des reliques forestières du Tonkpi 


\section{Richesse spécifique et distribution spatiale des primates dans la forêt classée de Krozialé}

Dans cette forêt, 301 indices de présence des primates ont été enregistrés. Parmi ces indices, $74 \%(\mathrm{~N}=223)$ sont directs et $26 \%(\mathrm{~N}=78)$ indirects. Les résultats de la prospection ont permis de recenser cinq espèces de primates dans la forêt classée de Krozialé. Elles sont représentées par le Galago de Thomas (Galagoïdes thomasi) avec 46,20\% ( $\mathrm{N}=139)$, Le Potto de Bosman (Perodictitus potto) 20,56\% ( $\mathrm{N}=62)$, le Cercopithèque de Campbell (Cercopithecus campbelli) 15,61\% ( $\mathrm{N}=47)$ et le Cercopithèque blanc-nez (Cercopithecus petaurista) 10,65\% ( $\mathrm{N}=32$ ). Le Colobe rouge (Piliocolobus badius) représente 6,98\% $(\mathrm{N}=21)$ des observations (Tableau I).

Tableau I : Espèces de primates inventoriées dans la forêt classée de Krozialé

\begin{tabular}{ccccc}
\hline Nom local & Nom Commun & Nom scientifique & Effectifs & Fréquence \\
\hline Foue & Cercopithèque de Campbell & Cercopithecus campbelli & 47 & 15,61 \\
Kpoun & Cercopithèque blanc-nez & Cercopithecus petaurista & 32 & 10,65 \\
Zèguè & Galago de Thomas & Galagoïdes thomasi & 139 & 46,20 \\
Nouin & Colobe rouge & Piliocolobus badius & 21 & 6,98 \\
Zon & Potto de Bosman & Perodictitus potto & 62 & 20,56 \\
\hline
\end{tabular}

Les investigations sur le terrain ont montré que les primates se rencontrent dans les périphéries Nord, Sud, Est et Ouest de la forêt classée de Krozialé. Galagoïdes thomasi et Perodictitus potto ont été les plus observée dans ces quatre zones de cette forêt. Cependant, Galagoïdes thomasi a été l'espèce la plus inventoriée à l'Ouest de la forêt classée de Krozialé. Concernant les autres espèces, Cercopithecus campbelli et Cercopithecus petaurista ont été plus observées à l'Est, au Nord et au Sud avec une abondance numérique d'indices de présence supérieure à celle de Piliocolobus badius. (Figure 3). 


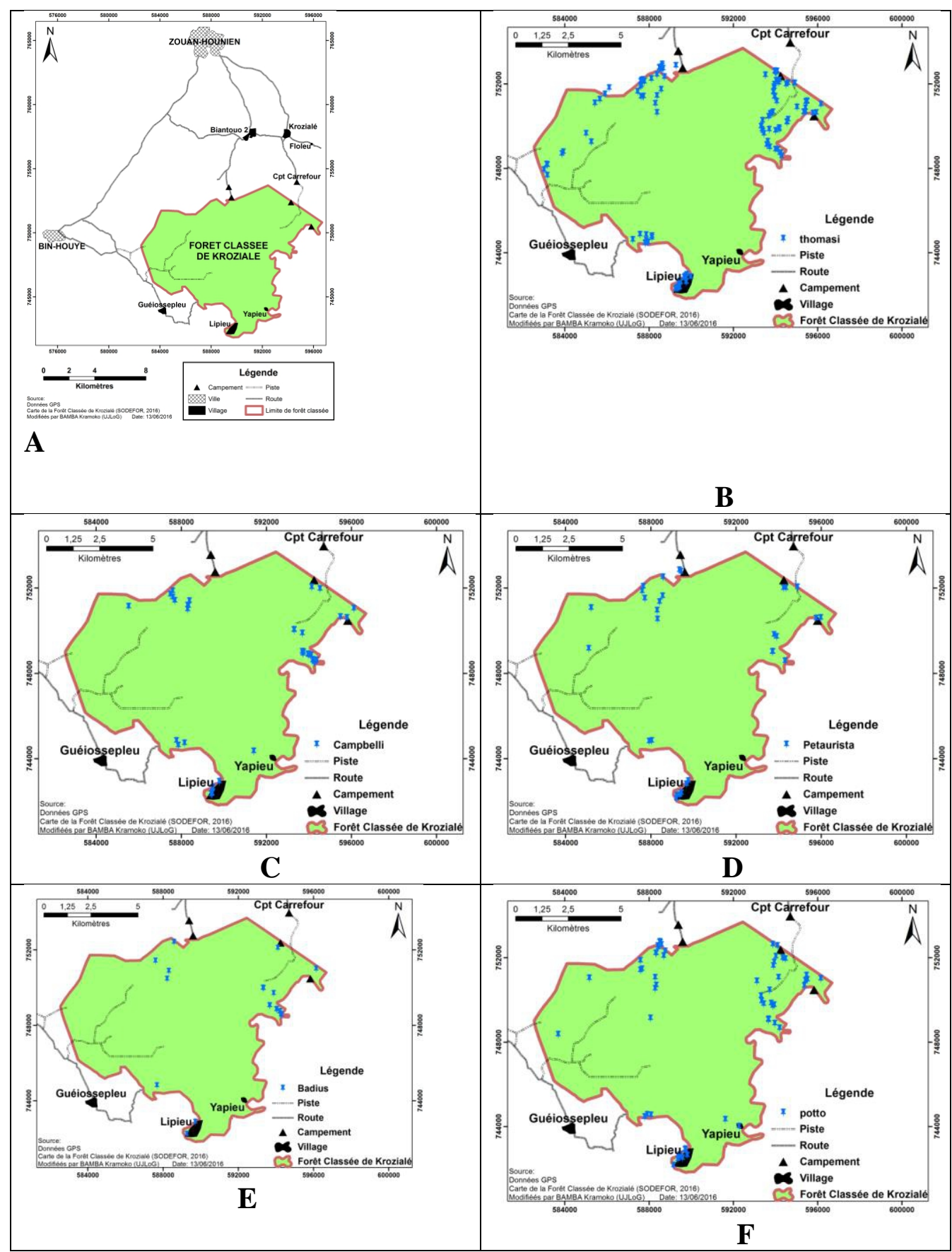

A = forêt classée de Krozialé en vert; distribution spatiale de Galagoïdes thomasi en B, Cercopithecus campbelli en C, Cercopithecus petaurista en D, Piliocolobus badius en E, Perodictitus potto en F.

Figure 3: Distribution spatiale des primates dans la forêt classée de Krozialé 


\section{Richesse spécifique et distribution des primates dans la forêt classée de Goulaleu}

Un total de 370 indices de présence de primate a été observé dans la forêt classée de Goulaleu dont 73,78\% sont d'observations directes $(\mathrm{N}=$ $273)$ et $26,22 \%$ indirectes $(\mathrm{N}=97)$. Six espèces de primates ont été inventoriées dans cette forêt. La plus abondante est le Galago de Thomas (Galagoïdes thomasi) avec 45,93\% ( $\mathrm{N}=170)$. Elle est suivie par le Potto de Bosman (Perodictitus potto) avec 17,86\% $(\mathrm{N}=66)$. Le Cercopithèque blancnez (Cercopithecus petaurista) et le Cercopithèque de Campbell (Cercopithecus campbelli) ont respectivement 13,51\% ( $\mathrm{N}=50$ ) et $10,54 \%$ $(\mathrm{N}=39)$. Le Callitriche (Cercopithecus sabaeus) a enregistré 7,57\% ( $\mathrm{N}=$ 28) des rencontres tandis que le Colobe blanc et noir (Colobus polykomos) a une fréquence de 4,59\% $(\mathrm{N}=17)$ (Tableau II).

Tableau II : Espèces de primates inventoriées dans la forêt classée de Goulaleu

\begin{tabular}{ccccc}
\hline Nom local & Nom Commun & Nom scientifique & Effectifs & Fréquence \\
\hline Gha & Colobe blanc et noir & Colobus polykomos & 17 & 4,59 \\
Zon & Potto de Bosman & Perodictitus potto & 66 & 17,86 \\
Foue & Cercopithèque de Campbell & Cercopithecus campbelli & 39 & 10,54 \\
Kpoun & Cercopithèque blanc-nez & Cercopithecus petaurista & 50 & 13,51 \\
Kletin & Callitriche & Cercopithecus sabaeus & 28 & 7,57 \\
Zèguè & Galago de Thomas & Galagoïdes thomasi & 170 & 45,93 \\
\hline
\end{tabular}

L'espèce de primates la plus observée dans la forêt classée de Goulaleu est Galagoïdes thomasi. Elle a été pratiquement observée sur toute la surface de la forêt. Il en est de même pour Perodictitus potto, mais avec une abondance numérique plus faible que celle de Galagoïdes thomasi. Cercopithecus petaurista, Cercopithecus campbelli, et Cercopithecus sabaeus ont été à majorité observées au centre et en périphérie de la forêt avec une abondance de Cercopithecus petaurista par rapport à Cercopithecus campbelli et Cercopithecus sabaeus. Colobus polykomos a aussi été inventoriée au centre et en périphérie de la forêt avec une abondance plus faible par rapport à celles des autres espèces de primate (Figure 4). 


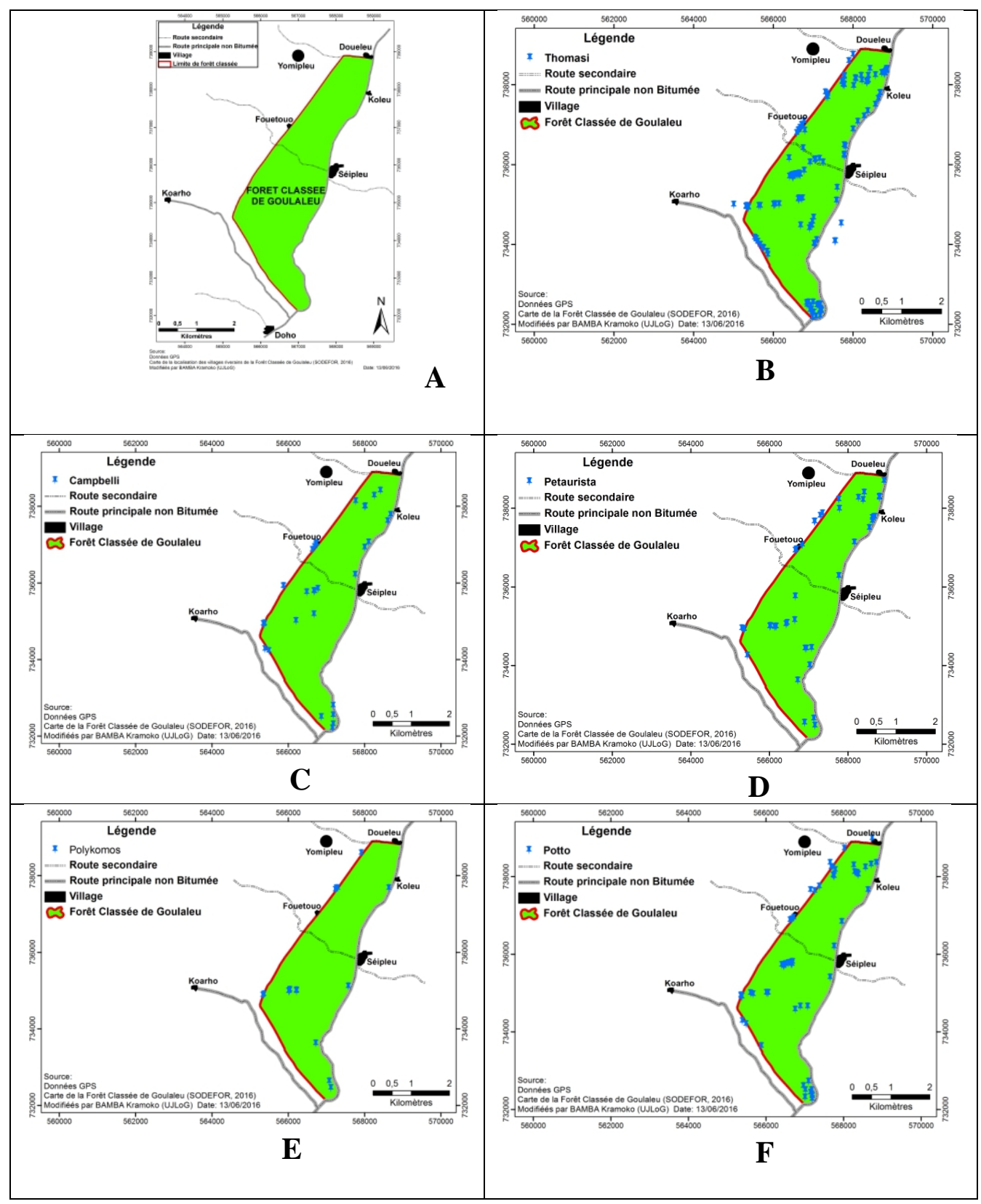




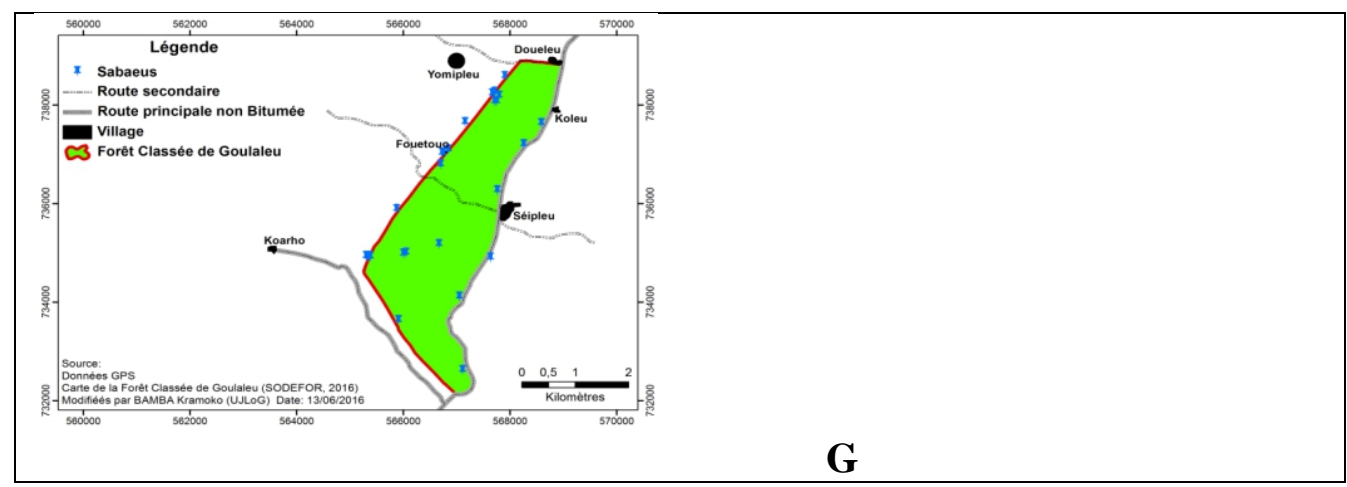

A = forêt classée de Goulaleu en vert; distribution spatiale de Galagoïdes thomasi en B, Cercopithecus campbelli en C, Cercopithecus petaurista en D, Colobus polykomos en E, Perodictitus potto en F, Cercopithecus sabaeus en G.

Figure 4: Distribution spatiale des primates dans la forêt classée de Goulaleu

\section{Richesse spécifique et distribution spatiale des primates dans la forêt villageoise}

Dans la forêt villageoise, il y a eu aussi des observations directes $(71,56 \% ; \mathrm{N}=146)$ et des observations indirectes $(28,44 \% ; \mathrm{N}=60) \mathrm{de}$ primate. Les prospections dans cette forêt ont permis de recenser cinq espèces de primates. Celle qui a été la plus observée est Galago de Thomas (Galagoïdes thomasi) avec $60,8 \%$ des observations $(\mathrm{N}=124)$. Ensuite viennent le Cercopithèque de Campbell (Cercopithecus campbelli) 12,74\% $(\mathrm{N}=26)$, le Potto de Bosman (Perodictitus potto) $12,25 \%(\mathrm{~N}=25)$ et le Cercopithèque blanc-nez (Cercopithecus petaurista) 9,80\% ( $\mathrm{N}=20)$. Une espèce de Colobe a été recensée dans cette forêt. Il s'agit du Colobe rouge (Piliocolobus badius) avec 9,80\% ( $\mathrm{N}=9$ ) (Tableau III).

Tableau III : Espèces de primates inventoriées dans la forêt villageoise

\begin{tabular}{ccccc}
\hline Nom local & Nom Commun & Nom scientifique & Effectifs & Fréquence \\
\hline Zon & Potto de Bosman & Perodictitus potto & 25 & 12,25 \\
Nouin & Colobe rouge & Piliocolobus badius & 9 & 4,41 \\
Foue & Cercopithèque de Campbell & Cercopithecus campbelli & 26 & 12,74 \\
Kpoun & Cercopithèque blanc-nez & Cercopithecus petaurista & 20 & 9,80 \\
Zèguè & Galago de Thomas & Galagoïdes thomasi & 124 & 60,8 \\
\hline
\end{tabular}

Les investigations ont montré que Galagoïdes thomasi est très abondante dans le Sud-ouest et le Sud-est de cette forêt villageoise par rapport aux autres espèces de primates. Elle a été la seule espèce observée dans le Nord de la forêt. Perodictitus potto, Cercopithecus campbelli, Cercopithecus petaurista ont été observées pratiquement avec les mêmes abondances dans le Sud-ouest et le Sud-est de la forêt. Piliocolobus badius a aussi été inventoriée dans les zones de distribution de Cercopithecus 
campbelli, Cercopithecus petaurista avec une abondance plus faible que celle de ces deux dernières espèces (Figure 5).

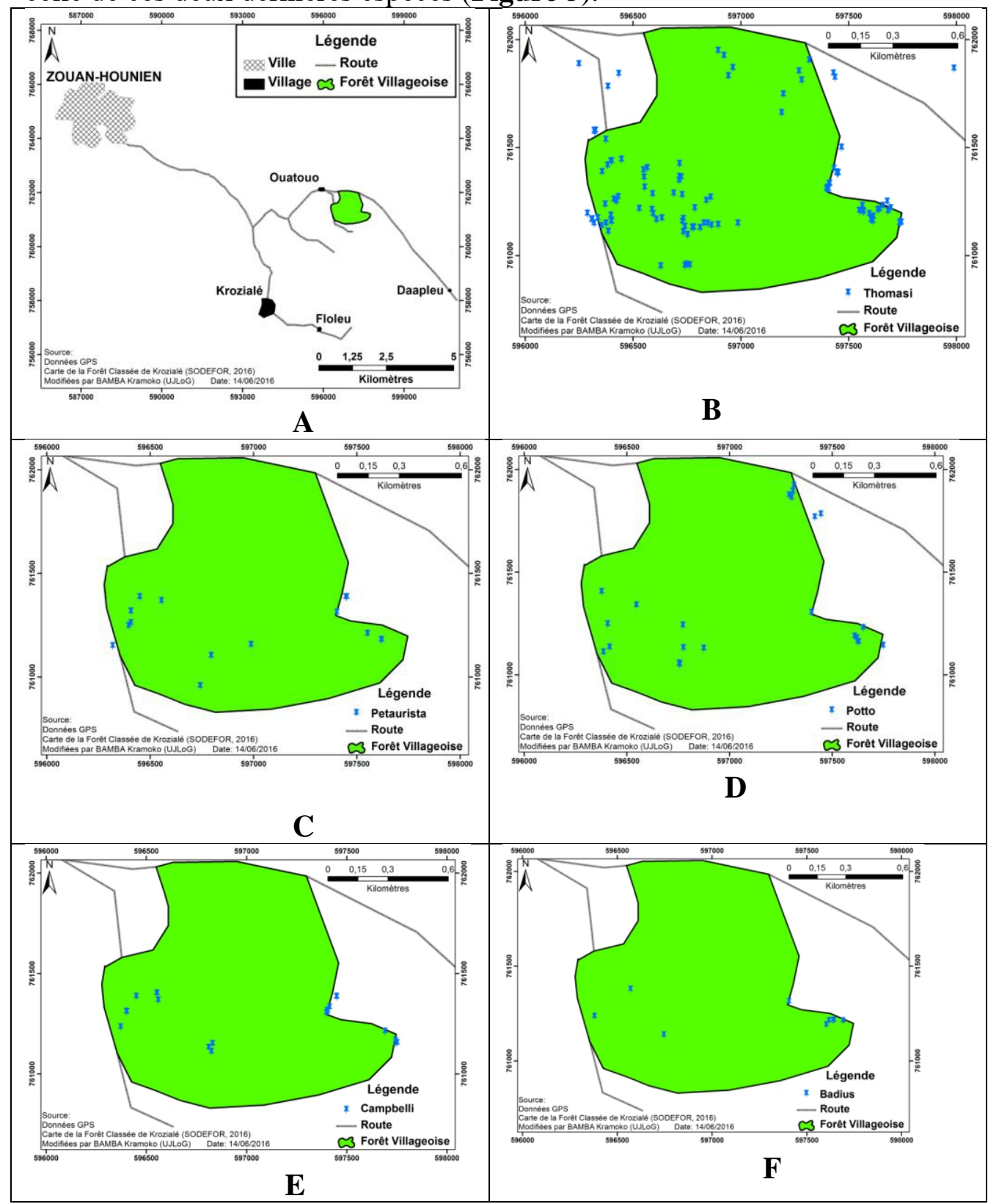

$\mathrm{A}=$ la forêt villageoise en vert; distribution spatiale de Galagoïdes thomasi en $\mathrm{B}$,

Cercopithecus petaurista en C, Perodictitus potto en D, Cercopithecus campbelli en E,

Piliocolobus badius en F.

Figure 5: Distribution spatiale des primates dans la forêt villageoise 


\section{Indices de diversité spécifique de primates dans les reliques de forêts}

La richesse spécifique en primates dans ces forêts a été évaluée grâce aux valeurs d'indice de Shannon. La forêt classée de Goulaleu présente l'indice de diversité de Shannon le plus élevée $(H=1,509)$. Ensuite vient la forêt classée de Krozialé avec un indice de diversité de Shannon de 1,396. L'indice de diversité le plus faible est enregistré dans la forêt villageoise avec une valeur de $\mathrm{H}=1,188$.

Quant à l'équitabilité, ses valeurs sont sensiblement égales dans les trois forêts. La forêt classée de krozialé a la plus grande valeur $(E=0,87)$, ensuite vient la forêt classée de Goulaleu $(\mathrm{E}=0,84)$ et enfin en forêt villageoise $(\mathrm{E}=0,74)$. (Tableau IV).

Tableau IV : Diversité d'espèce de primates par forêt prospectée Indices de présence

\begin{tabular}{ccccc}
\hline Espèce & FV & FC Krozialé & FC Goulaleu & Total \\
\hline Perodictitus potto & 25 & 62 & 66 & 153 \\
Galagoïdes thomasi & 124 & 139 & 170 & 433 \\
Cercopithecus sabaeus & 0 & 0 & 28 & 28 \\
Cercopithecus petaurista & 20 & 32 & 50 & 102 \\
Cercopithecus campbelli & 26 & 47 & 39 & 112 \\
Piliocolobus badius & 9 & 21 & 0 & 30 \\
Colobus polykomos & 0 & 0 & 17 & 17 \\
\hline Abondance numérique $(\mathrm{N})$ & $\mathrm{N}=204$ & $\mathrm{~N}=301$ & $\mathrm{~N}=370$ & $\mathrm{~N}=875$ \\
Nombre d'espèce $(\mathrm{S})$ & $\mathrm{S}=5$ & $\mathrm{~S}=5$ & $\mathrm{~S}=6$ & \\
Indice de Shannon $(\mathrm{H})$ & $\mathrm{H}=1,188$ & $\mathrm{H}=1,509$ & $\mathrm{H}=1,396$ & \\
Equitabilité & $\mathrm{E}=0,74$ & $\mathrm{E}=0,84$ & $\mathrm{E}=0,87$ & \\
\hline
\end{tabular}

$\mathrm{FV}=$ Forêt Villageoise $; \mathrm{FC}=$ Forêt Classée

Menaces anthropiques et statuts de conservation des primates dans la région du Tonkpi

\section{$\checkmark \quad$ Menaces anthropiques}

Au cours de cette étude, des indices $(\mathrm{N})$ d'activités anthropiques ont été recensés dans toutes les trois reliques forestières. Il s'agit de l'agriculture $(65,42 \% ; \mathrm{N}=931)$, la chasse $(12,22 \% ; \mathrm{N}=174)$, l'orpaillage $(10,32 \% ; \mathrm{N}$ $=147)$, l'exploitation forestière $(7,72 \% ; \mathrm{N}=110)$ et l'exploitation minière $(4,32 \% ; \mathrm{N}=62)$. Les fréquences de rencontre de ces différents indices varient d'une forêt à une autre :

$\mathrm{Au}$ sein de la forêt classée de Goulaleu, la diversité des primates est menacée à $69,67 \%(\mathrm{~N}=342)$ par l'agriculture. L'exploitation forestière a une fréquence de rencontre de $12,22 \%(\mathrm{~N}=60)$. Dans cette forêt, l'orpaillage se pratique avec $9,36 \%(\mathrm{~N}=46)$ quand les indices de chasse ont une fréquence de $8,75 \%(\mathrm{~N}=43)$. Aucun indice d'exploitation minière n'a été enregistré dans cette forêt classée. 
Dans la forêt classée de Krozialé aussi, l'agriculture est l'activité humaine la plus pratiquée avec $60,47 \%(\mathrm{~N}=326)$ des observations. Ensuite vient l'orpaillage avec une fréquence de $17,46 \%(\mathrm{~N}=94)$. Les indices de chasse ont une fréquence de $9,46 \%(\mathrm{~N}=51)$ et la fréquence de prélèvement du bois dans la forêt classée de Krozialé est de 8,72\% ( $\mathrm{N}=47)$. Les sondages effectués par "Persus mining Côte d'Ivoire SA » qui serais venu avec une autorisation gouvernementale selon nos renseignements auprès du capitaine Ebé Yao Bertin (chef U.G.F Danané) ont une fréquence de $3,89 \%(\mathrm{~N}=21)$.

En forêt villageoise, c'est toujours l'agriculture qui domine les activités anthropiques. Sa fréquence est de 66,76\% ( $\mathrm{N}=263)$. La chasse arrive en deuxième position avec une fréquence de $20,30 \%(\mathrm{~N}=80)$. Dans cette forêt villageoise, la Société des Mines d'Ity (SMI) a déjà réalisé des sondages en vue de son exploitation. La fréquence de rencontre d'indices des activités de cette société s'élève à $10,40 \%(\mathrm{~N}=41)$. Quant à l'orpaillage, il est représenté à $1,78 \%(\mathrm{~N}=7)$. Le prélèvement du bois y est très faible et ne représente que $0,76 \%(\mathrm{~N}=3)$ des cas.

Selon le test de Kruskal-Wallis, toutes ces activités anthropiques constituent pratiquement un même niveau de menace sur la diversité des primates $(\mathrm{P}$ value $=0,99>0,05)$ (Figure 6).

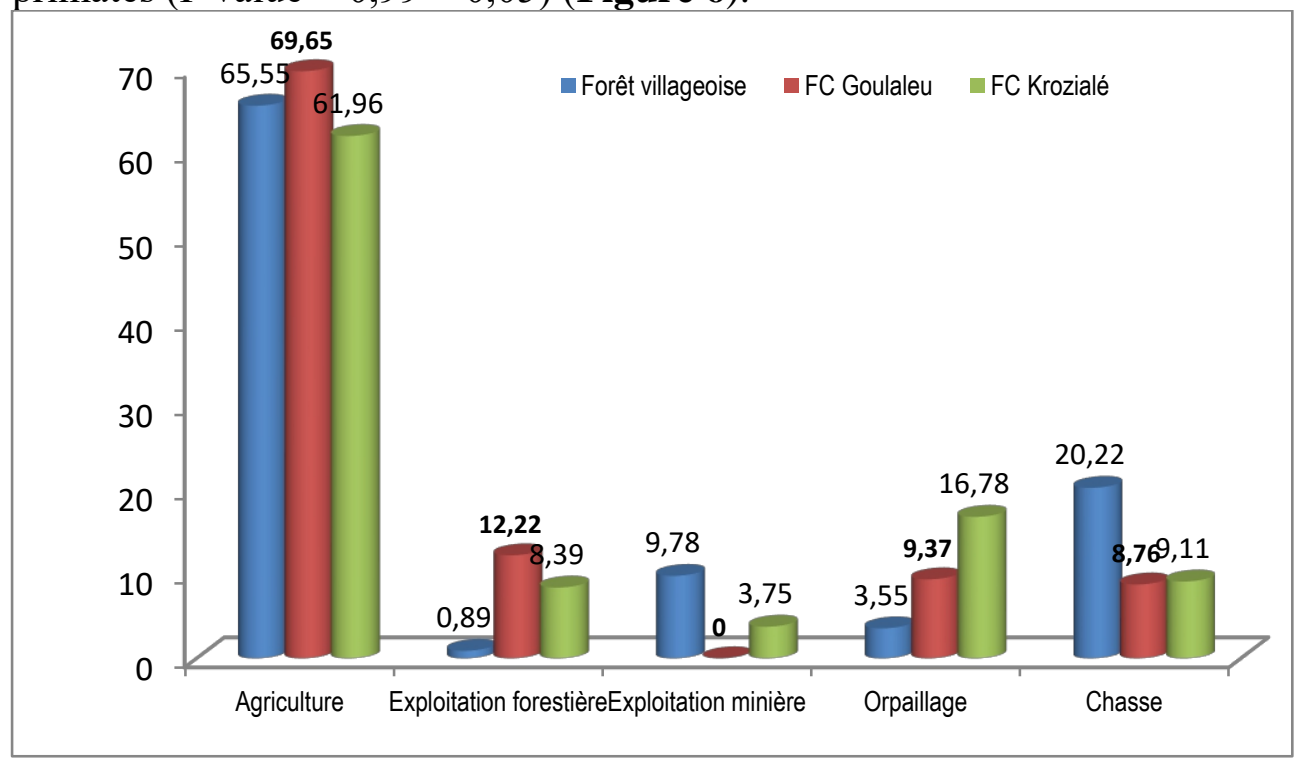

Figure 6 : Menaces sur les primates dans la région du Tonkpi

\section{$\checkmark \quad$ Statut de conservation des primates de la région du Tonkpi}

Sept espèces de primates ont été inventoriées au cours de cette étude. Selon la classification de l'UICN 2016, une espèce est en danger. Il s'agit du Colobe rouge (Piliocolobus badius). Le Colobe blanc et noir (Colobus polykomos) est vulnérable aux activités anthropiques. Le Cercopithèque de 
Campbell (Cercopithecus campbelli), le Callitriche (Cercopithecus sabaeus) le Cercopithèque blanc-nez (Cercopithecus petaurista), le potto de Bosman (Perodictitus potto) et le Galago de Thomas (Galagoïdes thomasi) appartiennent à la catégorie des espèces à préoccupation mineure.

En se basant sur l'estimation des abondances donnée par les populations riveraines au cours des enquêtes, nous avons évalué le statut de conservation locale de ces primates. Ainsi, Galagoïdes thomasi est très abondante dans la région du Tonkpi. Les espèces comme Cercopithecus campbelli, Cercopithecus petaurista, Cercopithecus sabaeus et Perodictitus potto, sont abondantes. Certaines se font rares. Il s'agit de Colobus polykomos, et Piliocolobus badius (Tableau IV).

Tableau IV : Statut de conservation des primates de la région du Tonkpi

\begin{tabular}{cccc}
\hline Espèces & \multicolumn{3}{c}{ Statut de conservation } \\
\hline Non Scientifique & Fréquence & Statut local & Statut UICN \\
\hline Cercopithecus campbelli & 12,80 & ++ & LC \\
Cercopithecus petaurista & 11,66 & ++ & LC \\
Cercopithecus sabaeus & 3,2 & ++ & LC \\
Colobus polykomos & 1,94 & + & VU \\
Galagoïdes thomasi & 49,44 & +++ & LC \\
Perodictitus potto & 17,48 & ++ & LC \\
Piliocolobus badius & 3,48 & + & EN \\
\hline
\end{tabular}

$(+++=$ très abondant $) ;(++=$ abondant $) ;(+=$ peu abondant $)$;

$\mathrm{EN}=$ en danger $) ;(\mathrm{LC}=$ préoccupation mineur $) ;(\mathrm{VU}=$ vulnérable $)$

\section{Discussion}

Certaines forêts dont les forêts classées font objet de protection sur le territoire ivoirien (Bitty et al., 2013). La Côte d'Ivoire compte 231 forêts classées qui en plus de l'épuration de l'air jouent un rôle important dans la conservation durable des primates (Assoa, 2004 ; Tieha, 2010). Parmi ces forêts classées, deux (les forêts classées de Krozialé et Goulaleu) ont fait objet d'inventaire de primates dans la région du Tonkpi, en plus d'une forêt située dans le domaine villageois. Sept espèces de primates ont été inventoriées dans ces reliques de forêts. La diversité spécifique des primates varie d'une forêt à l'autre.

Dans ces trois forêts, les valeurs d'indice de Shannon oscillent entre 1,188 et 1,509. La valeur de 1,509 enregistrée dans la forêt classée de Krozialé serait due à la présence du fleuve Cavally et de vastes étendues de zones inondées. C'est dans ces zones inondées, qu'a été observé le Colobe rouge (Piliocolobus badius). Ainsi, les forêts inondées qui échappent aux activités anthropiques, à cause justement de la difficulté à y accéder, constituent de plus en plus les seuls refuges pour certaines espèces de primates, dans les régions qui ont perdu une grande partie de leur couverture 
forestière. Ce type de fragments forestiers comme la forêt des marais Tanoé, au sud-est de la Côte d'Ivoire, deviennent donc très importants pour la conservation des primates menacés de disparition (Béné et al., 2012).

La faible valeur d'indice de Shannon dans la forêt villageoise par rapport à celles des forêts classées s'expliquerait par le fait qu'elle ne fait l'objet d'aucune protection. Mais en termes de conservation de primates, la valeur d'indice de Shannon dans la forêt villageoise est sensiblement égale à celles des forêts classées. Ce résultat illustre le fait qu'en plus des forêts classées, certaines forêts villageoises continuent à jouer un grand rôle dans la conservation durable des primates dans la région du Tonkpi. En 2004, Lamon a mis en relief l'importance de l'aménagement des forêts villageoises en périphérie du parc du $\mathrm{W}$ en vue d'une conservation durable de la faune sauvage au Burkina Faso. De plus, pour une conservation durable des aires protégées, les forêts villageoises bien conservées sont indispensables et doivent être donc intégrées dans les plans de gestions de la faune sauvage sur le territoire ivoirien (Kouakou, 2014). Les valeurs d'équitabilité, sensiblement égales entre les trois forêts indiquent qu'aucune espèce ne domine largement le peuplement, mais quelques espèces sont Codominantes.

C'est ainsi que ces espèces présentent des similitudes et des différences dans leurs distributions spatiales au sein de chaque forêt. Dans la forêt classée de Krozialé, Cercopithecus campbelli, Perodictitus Potto, Cercopithecus petaurista et Piliocolobus badius s'observent au Nord, au Nord-est et au Sud de la forêt. En plus des zones déjà mentionnées, Galagoïdes thomasi s'observe aussi à l'Est de cette forêt. La présence du fleuve Cavally expliquerait la distribution d'un grand nombre de primates au Nord-est de la forêt.

Contrairement à la forêt classée de Krozialé, celle de Goulaleu est parsemée de plusieurs montagnes qui interviennent dans la distribution spatiale des primates. En effet, la majorité des primates a été observée dans les forêts au sommet des montagnes situées en périphérie de la forêt. Les montagnes jouent un rôle important dans la conservation des primates dans la région du Tonkpi (Galat-Luong \& Gérard, 2000). Des observations ont aussi eu lieu dans les zones inondées (Béné et al., 2007 ; Béné et al., 2012) en bordure de l'axe Goulaleu-Toulepleu. Les espèces de primates s'observent pour la plupart en périphérie de la forêt classée de Goulaleu. Mais les prosimiens (Galagoïdes thomasi et Perodictitus Potto) sont peu abondants à l'intérieur de la forêt classée de Goulaleu.

A l'image de la forêt classée de Goulaleu, la forêt villageoise a en son sein une montagne et aussi une zone inondée. La montagne est située au Sudouest tandis que la zone inondée se trouve au Sud-est de la forêt. L'observation des singes au Sud-ouest et au Sud-est de la forêt villageoise 
serait due aux présences respectives de la montagne et de la zone inondée (Béné et al., 2012). La distribution spatiale des prosimiens ne respecte pas cette configuration. En effet, en plus du Sud-ouest et du Sud-est, le Perodictitus potto s'observe aussi au Nord-est de la forêt. Galagoïdes thomasi est pratiquement présente sur toute la surface de la forêt.

Galagoïdes thomasi est l'espèce de primates la plus abondante dans ces trois forêts prospectées. Il s'observe aussi bien en périphérie qu'à l'intérieur des forêts. Sa forte représentativité serait due à son faible poids (Jonathan, 2006) qui ne fait pas de lui un véritable butin de chasse (Caspary et al., 2001 ; Roger et al., 2011). Quant aux Colobes, ils ont une abondance numérique très faible dans les trois forêts. Cette rareté des Colobes dans ces forêt serait due au fait qu'elles sont très vulnérables à la chasse (Koné, 2004 ; Béné et al., 2013). Des études ont montré que les colobes rouges sont fortement vulnérables à la chasse car ne sont pas très doués pour détecter les chasseurs et prédateurs (Bshary et Noé, 1997). Une fois qu'un chasseur repère un groupe de colobes rouges, ces singes se montrent souvent curieux ou parfois même agressifs. Ils adoptent rarement une stratégie de "se-cacheret-attendre" comme d'autres singes le font, faisant d'eux des cibles faciles (Lieven, 2015). L'observation des espèces de primates à la périphérie des trois forêts s'expliquerait aussi par la présence des activités anthropiques à l'intérieur de ces forêts. En effet les populations riveraines des reliques de forêts investissent l'intérieur des forêts, laissant intactes leurs périphéries pour tromper la vigilance des autorités. Ces périphéries encore intactes seraient favorables à la survie des primates.

Toutes ces espèces de primate inventoriées dans les trois reliques de forêt n'ont pas le même statut de conservation, au plan local comme international. C'est ainsi que sur le plan international, une espèce est classée en Danger (Piliocolobus badius), une autre est dans la catégorie Vulnérable (Colobus polykomos) et cinq relèvent de la catégorie Préoccupation mineur (Galagoïdes thomasi, Perodictitus Potto, Cercopithecus campbelli, Cercopithecus petaurista et Cercopithecus sabaeus) (UICN, 2016). Au plan local, les abondances relatives selon les enquêtes font état de ce que, Galagoïdes thomasi est très abondant dans la région du Tonkpi. Les espèces que sont : Cercopithecus campbelli, Cercopithecus petaurista, Cercopithecus sabaeus et Perodictitus potto, sont abondantes. Certaines espèces sont peu abondantes. Il s'agit de Colobus polykomos, et Piliocolobus badius. Ce statut de conservation local d'espèces de primate dans la région du Tonkpi a déjà été confirmé lors de ses études à l'intérieur et à l'extérieur de la réserve intégral du mont nimba (Kouakou, 2014), et les raisons sont multiples.

Parmi les causes de la disparition des primates, l'action anthropique est de loin la plus en vue (Zadou et al., 20011). Au cours de cette étude, divers indices ont indiqué la présence d'activité humaines dans les forêts. Il 
s'agit des champs (activité agricole), d'exploitations forestières et minières, d'orpaillage et de chasse. La présence humaine régulière dans ces forêts pourrait troubler la quiétude des primates et augmenter leur intensité de prélèvement. Ces indices d'activité anthropique se répartissent sur toutes les étendues des forêts classées, alors que dans ces espaces, les activités des populations riveraines devraient se limiter au ramassage du bois mort, à la cueillette des fruits, et au prélèvement des plantes alimentaires et médicinales (Zeta \& Kragbé, 2012 ; Jen, 2012). Les singes auraient donc pu s'y développer aisément si les infiltrations ne concernaient pas les facteurs les plus menaçants que sont le braconnage et l'agriculture à travers la déforestation (Béné et al., 2012). Cette déforestation du fait de l'augmentation des surfaces agricoles est une réalité dans le monde entier (COMIFAC, 2005 ; Xavier et al., 2008 ; Moussa et al., 2014). Les indices de braconnage sont légions dans la forêt villageoise mais aussi dans les forêts classées. Les outils de chasse ne sont pas sélectif donc ne permettent pas une gestion durable de la faune sauvage (Béné et al., 2013). L'exploitation forestière a aussi de nombreuses conséquences néfastes sur les populations de primates. Elle accélère la diminution de leurs domaines vitaux, écourtant ainsi leurs expériences de vie. A Lopé, au Gabon, White (1994) a montré que les groupes de chimpanzés qui ont été chassés de leur territoire par les exploitations forestières, ont eu des bagarres mortelles avec d'autres groupes de chimpanzés lorsqu'ils essayaient de s'installer sur un territoire déjà occupé. De plus, cette activité a pour corolaire, l'ouverture de pistes permettant aux paysans et autres chasseurs d'accéder à des zones impossibles auparavant (Zeta \& Kragbé, 2012 ; Jen, 2012). L'exploitation minière et l'orpaillage sont également des activités dangereuses pour les primates (Bebbington \& Williams, 2008). En plus de participer à la destruction de leurs habitats, les produits utilisés dans cette activité comme le mercure se retrouvent dans les cours d'eaux. Une fois ingérés par les singes qui s'abreuvent de cette source d'eau, ces produits peuvent provoquer des troubles neurologiques (Bwenda, 2013 ; ISF 2016).

\section{Conclusion}

A l'issue de cette étude, sept (7) espèces de primates ont été inventoriées dans trois reliques forestières dans la région du Tonkpi à l'Ouest de la Côte d'Ivoire. Dans ces forêts, vivent deux espèces de primates inscrites sur la liste rouge des espèces protégées de l'Union Internationale pour la Conservation de la Nature (UICN). Ce sont : le Colobe blanc et noir (Colobus polykomos) et le Colobe rouge (Piliocolobus badius). La présence dans ces reliques forestières d'espèces de primates dont la protection est d'intérêt mondial démontre leur valeur pour la conservation durable des primates. Cependant, la pratique de l'agriculture, l'exploitation forestière, 
l'orpaillage, l'exploitation minière et la chasse, dans ces forêts laissent planer un doute sur le maintien et la survie de ces animaux. L'état ivoirien doit donc prendre des mesures d'atténuation des impacts négatifs des activités anthropiques pour la sauvegarde de ces forêts.

\section{References:}

1. Assoa, A., 2004, Stratégie de gestion durable des éléphants en Côte d'Ivoire, programme 2005-2014, $100 \mathrm{p}$.

2. Buckland, S.T., Anderson, D.R., Burnham K.P., \& Laake, J.L. 1993, Distance Sampling. Estimating Abundance of Biological Populations, London, Chapman and Hall, 1st ed., 446 p.

3. Bebbington, A., \& M. Williams, 2008, Water and Mining Conflicts in Peru."Mountain Research and Development. 28(3), pp. 190-195.

4. Béné, J-C. K., Inza, K., \& Klaus, Z. 2007, Répertoire et contextes sociaux des cris unitaires du colobe vert (Procolobus verus) dans le parc national de Taï (PNT), Côte d'ivoire, 4(2), pp. 137-147.

5. Béné, J.C. K., Koné, I., Gonédélé Bi, S., Bitty, E. A., Ouattara, K., Akpatou, K. B., N'Guessan, K. A., \& Koffi, D. A. 2012, The diurnal primate community of the Tanoé Forest: species composition, relative abundance, distribution, polyspecific associations and conservation status Int. J. Biol. Chem. Sci, 6(1), pp. 51-64.

6. Béné, J-C. K., Daouda, D., \& Eric, N.2015, Gestion durable de la faune et des ressources cynégétiques en Côte d'Ivoire. Rapport pour les Etats généraux de la forêt, de la faune et des ressources en eau, $101 \mathrm{p}$.

7. Bitty, E. A., Kadjo, B., Gonedelé bi, S.B., Okon, M.O., \& Kouassi, P.K., 2013, Inventaire de la faune mammalogique d'une forêt urbaine, le Parc National du Banco, Côte d'Ivoire. International journal of Biological and Chemical Sciences, 7(4), pp. 1678-1687.

8. Bwenda, 2013, L'exploitation minière, une menace pour les aires protégées du Katanga cas de phelps dodge Congo à la basse Kando, $24 \mathrm{p}$.

9. Caspary, H.U., Koné, I., Prouot, C., \& De Pauw, M.2001, La chasse et la filière viande de brousse dans l'espace Taï, Côte-d'Ivoire. Tropenbos -Côte-d'Ivoire séries 2, $98 \mathrm{p}$.

10. CNRA, 2009, La direction régionale du CNRA de Man en quelques mots et chiffres, $12 \mathrm{p}$.

11. COMIFAC, 2005, Les forêts du bassin du Congo évaluation préliminaire, $39 \mathrm{p}$.

12. Dieulin, 2008, L'analyse des données climatiques en Côte d'Ivoire 20 p. 
13. Étienne, S., \& François, D. 2012, Chasse \& biodiversité. Rapport du RAC sur les conséquences de la chasse sur la faune, $16 \mathrm{p}$.

14. GALAT-LUONG, A., \& Gérard, G. 2000, Les primates des monts nimba, 21p.

15. Hoppe-Dominik, B., Kühl, H.S., Radl G., \& Fischer, F. 2011, Longterm monitoring of large rainforest mammals in the biosphere reserve of Taï National Park, Côte d'Ivoire. African Journal of Ecology, 49 (4), pp. 450-458.

16. ISF, 2016, Etat des lieux des conséquences graves de l'exploitation minières, $26 \mathrm{p}$.

17. Jen, D., 2012, Exposition “Amazonie: pourquoi tant de biodiversité? $14 \mathrm{p}$.

18. Jonathan, K., 2006, Guide des Mammifères d'Afrique, 272 p.

19. Kadjo, B., Azani, D., Tsague, L., \& Gomse, A. 2014, Etat des lieux des populations d'Hippopotames et autres grands mammifères du Parc National de la Marahoué (Côte d'Ivoire). Agronomie Africaine, 26 (2), pp. 89 - 101.

20. Koné, I., 2004, Effet du braconnage sur quelques aspects du comportement du colobe bai Procolobus [piliocolobus] badius et du cercopitèque diane Cercopithecus diana diana dans le Parc National de Taï, Côte d'Ivoire, Thèse de Doctorat, Université de Cocody, Abidjan, $146 \mathrm{p}$.

21. Koné, I., Joanna, E. L., Johannes, R., Adama, B. 2008, Primate seed dispersal and its potential role in maintaining useful tree species in the Taï region, Côte-d'Ivoire: implications for the conservation of forest fragments, 1(3), pp. 293-306

22. Koné, M., Konate, S., Yéo, K., Kouassi, K. P., Linsenmair, K.E. 2014, Effects of Management intensity on ant diversity in cocoa plantation (Oume, Centre west Côte d'Ivoire). Journal of Insect Conservation, 18(4), pp. 701-712

23. Kouakou, H.B., 2014, Action pour la sauvegarde de la biodiversité de la réserve naturelle intégrale du Mont Nimba, Côte d'Ivoire : Evaluation de la diversité faunistique et campagne de sensibilisation, $18 \mathrm{p}$.

24. Lamon, A., 2004, Gestion communautaire de la grande faune et aménagement des terroirs villageois en périphérie du Parc du $\mathrm{W}$ au Burkina Faso. Mémoire de fin d'étude, Faculté Universitaire des Sciences Agronomiques de Gembloux, 82 p.

25. Lieven, D., 2015, Première photo au monde d'un singe considéré comme éteint au Congo 5p.

26. Moussa, K., Yao, L. K., Danho, F. R. N., Djah, F. M., \& Lacina, C.2014, Évolution de la couverture forestière de la Côte d'Ivoire des 
années 1960 au début du 21e siècle International Journal of Innovation and Applied Studies, 7(2), pp. 782-794

27. N'Guessan, F. K., Irié, A., \& ZORO, B. 2010, Nouveau découpage de la zone de forêt dense humide de la Côte d'Ivoire, 7(2), pp. 177194.

28. Roger, A. M., Henri, B., Christophe, N., Quevin, F. B., Innocent, N., Jean-Luc, H., Pascal, L., \& Cédric, V. 2011, Profil des vendeurs de viande de chasse et évaluation de la biomasse commercialisée dans les marchés municipaux de Brazzaville, Congo, 4(2), pp. 203-217.

29. Tieha, V., 2010, Ministère de l'Environnement, des Eaux et Forêts CI, Politique forestière 2010-2015, 135p.

30. UICN, 2015, IUCN Red List of Threatened Species, Version 2015, 2, [En ligne], URL : http:// www.iucnredlist.org, Consulté le 16 Septembre 2016.

31. White, 1994, Biomass of rain forest mammals in the Lope reserve, Gabon. J.Anim.Ecol, 63, pp. 499-512.

32. Walsh, P. D., \& White, L.J.T. 1999, What It Will Take to Monitor Forest Elephant Populations. Conservation Biology 13, pp. 11941202.

33. White, L., \& Edwards, A.2000, Conservation Research in the African Rain Forests: A Technical Handbook. New York, USA.

34. Xavier, L. R., Barbault, R., Baudry, J., Burel, F., Doussan, I., Garnier, E., Herzog, F., Lavorel, S., Lifran, R., Roger- Estrade, J., Sarthou, J. P., \& Trommetter, M. 2008,

Agriculture et biodiversité. Valoriser les synergies, 116p.

35. Yéo, K., Tiho, S., Ouattara, K., Konate, S., Kouakou, L.M.M., \& Fofana, M. 2013, Impact de la fragmentation et de la pression humaine sur la relique forestière de l'Université d'Abobo-Adjamé (Côte d'Ivoire). Journal of Applied Biosciences, 61, pp. 4551- 4565.

36. Zadou, D. A., Koné, I., Mouroufié, K.V., Adou-Yao, C. Y., Gléanou, K. E., Kablan, A.Y., Coulibaly, D., \& Ibo, G. J. 2011, La valeur de la forêt des Marais Tanoé-Ehy (Sud-Est de la Côte d'Ivoire) pour la conservation : dimension socio-anthropologique. Tropical Conservation Science, 4 (4), pp. 373-385.

37. ZETA, K. A., \& KRAGBE, A. G. 2012, La protection juridique du patrimoine forestier, 53p. 